\title{
Dynamical Asteroseismology: towards improving the theories of stellar structure and (tidal) evolution
}

\author{
Andrew Tkachenko ${ }^{1, \star}$ \\ ${ }^{1}$ Instituut voor Sterrenkunde, KU Leuven, Celestijnenlaan 200D, B-3001 Leuven, Belgium
}

\begin{abstract}
The potential of the dynamical asteroseismology, the research area that builds upon the synergies between the asteroseismology and binary stars research fields, is discussed in this manuscript. We touch upon the following topics: i) the mass discrepancy observed in intermediate- to high-mass main-sequence and evolved binaries as well as in the low mass systems that are still in the pre-main sequence phase of their evolution; ii) the rotationally induced mixing in high-mass stars, in particular how the most recent theoretical predictions and spectroscopic findings compare to the results of asteroseismic investigations; iii) internal gravity waves and their potential role in the evolution of binary star systems and surface nitrogen enrichment in high-mass stars; iv) the tidal evolution theory, in particular how its predictions of spin-orbit synchronisation and orbital circularisation compare to the present-day high-quality observations; v) the tidally-induced pulsations and their role in the angular momentum transport within binary star systems; vi) the scaling relations between fundamental and seismic properties of stars across the entire HR-diagram.
\end{abstract}

\section{Introduction}

Asteroseismology is a study of deep interiors of stars through interpretation of frequencies, amplitudes, and phases of their oscillation modes. Similar to the seismology of our own Earth, one makes use of the fact that different pulsation modes probe different layers inside a star, providing information about the physical conditions in the stellar interiors. Depending on their position in the Hertzspung-Russel (HR) diagram, stars are found to pulsate in acoustic (p-), gravity ( $\mathrm{g}-)$, or mixed modes. These latter oscillations have the properties of $\mathrm{g}$-modes near the stellar core while posses the p-mode character in the outer regions of a star. Pressure and gravity are the restoring forces for the acoustic and gravity waves, respectively. Pulsating stars occupy many different places in the HRdiagram making the assteroseismic studies possible for a variety of stellar objects in a wide range of stellar masses and at different evolutionary stages (see Fig. 1). A discussion of different types of pulsating stars as well as the wave excitation mechanisms is beyond the scope of this paper. We thus refer the interested reader to [1] for more details.

Asteroseismology has been revolutionized with the launch of space missions such as MOST [36], CoRoT [5], and Kepler [9], which delivered photometric data of unprecedented quality and allowed detailed studies of stellar objects across the whole HR-diagram. An observational probe of the near core regions, as well as the measurements of the internal rotation have recently become available for main-sequence (MS) stars of spectral types B to F [e.g., 22, 26, 33, 35]. Space-based data provided us with

\footnotetext{
^e-mail: Andrew.Tkachenko@ster.kuleuven.be
}

new insights into stellar objects at advanced evolutionary stages (e.g., RR Lyrae stars - [25]; subdwarf B-type stars - [8]), revolutionized our understanding of evolved red-giant stars exhibiting stochastically excited solar-like oscillations [e.g., 7], and significantly improved our understanding of stars during early, pre-main-sequence (preMS) stages of their evolution [43].

Despite the huge success of asteroseismology in the past decade, it still remains a model dependent research field, with the model dependency being significantly larger for intermediate- and high-mass stars than for sun-like stars. Insufficient quality of observations is no longer a limiting factor for a new series of progressions in asteroseismology, but the shortcomings in the current theories of stellar structure and evolution become a real bottleneck. Thanks to the fact that many pulsating stars are found in binary systems across the whole HR-diagram, binary stars - systems consisting of two stellar objects that periodically revolve around their common center of mass - can provide us with additional, model-independent observational constraints in terms of the dynamical masses and radii of stars. Nowadays, we talk about dynamical measurements of fundamental properties of stars in large mass and age ranges to precisions approaching $1 \%$ [e.g., 15, 31]. This way, asteroseismic investigations can be confronted/supplied with model-independent fundamental properties of stars, providing a valuable test of the current theories of stellar structure and evolution. In addition, pulsating stars in binary systems allow us to test and further develop theories of tidal evolution and angular momentum transport - both theories can be observationally tested and further refined thanks to asteroseismology delivering pre- 
cise observational constraints on the internal structure of stars. Dynamical tides are known to be effective in resonant excitation of stellar pulsations but magnetic fields in binaries are expected to strongly affect the transfer of mass and angular momentum as well as the global properties of stellar oscillations. The interplay between stellar magnetic fields, binarity, and oscillations has yet to be investigated in detail, and will provide even further insight into the tidal evolution theory. A few applications of the dynamical asteroseismology (the field that builds upon the synergies between asteroseismology and binary star research areas) as well as its potential impact on the stellar astrophysics as a whole are discussed in this manuscript.

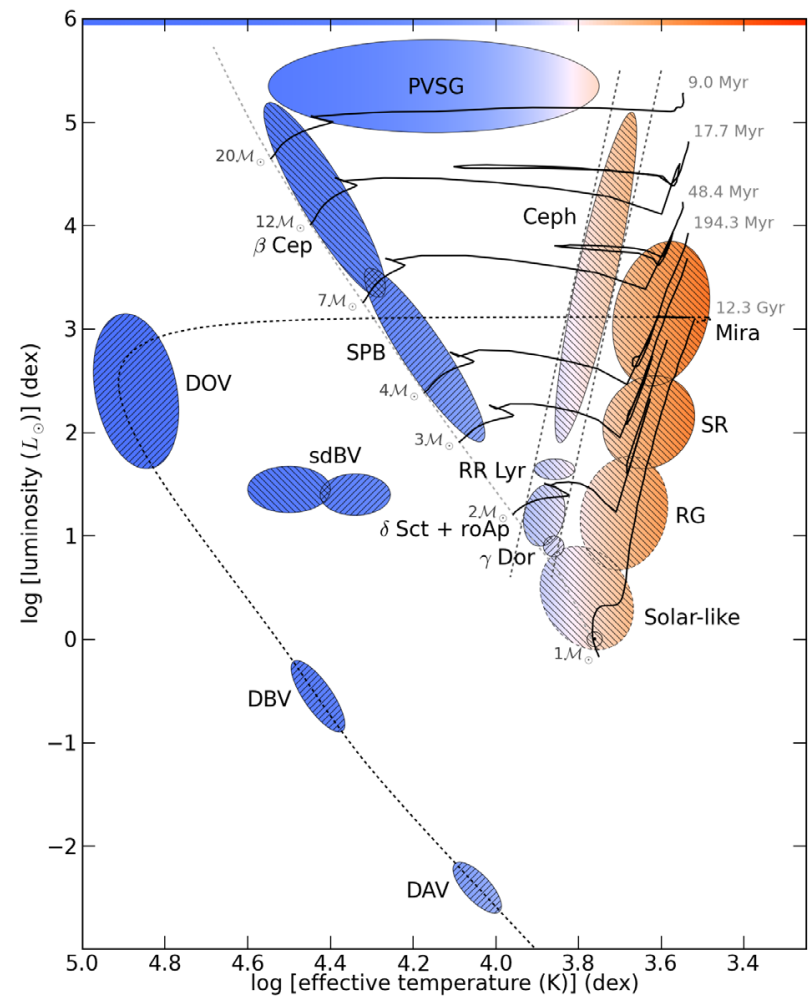

Figure 1: Pulsation HR-diagram showing different types of stars for which asteroseismic studies are possible. Colour coding (approximately) resembles temperature scale, with orange/blue indicating coolest/hottest stars. A few evolutionary tracks are overplotted to give a hint on the range of stellar masses. Image credit: Dr. P. I. Pápics.

\section{Mass discrepancy}

One of the problems that is currently pending a solution in the research field of massive binary stars is the mass discrepancy problem. In its original formulation, the term mass discrepancy refers to the disagreement between the spectroscopic and evolutionary masses of the star [17]. The evolutionary mass is determined by fitting evolutionary tracks to a position of the star in the HR-diagram, whereas the spectroscopic mass is computed from the spectroscopically derived value of the surface gravity and from the radius of the star. This original definition of the mass discrepancy has nothing to do specifically with binary systems but refers to massive stars in general. We keep on using the term mass discrepancy in application to binary systems throughout the manuscript, and warn the reader that we refer to the discrepancy between the evolutionary masses and those inferred from binary dynamics (dynamical masses).

The V380 Cyg binary system is probably one of the best illustrations of the mass discrepancy observed in massive binary stars. The system was originally investigated by [16] based on ground-based multicolour photometry and high-resolution spectroscopy. One of the main results obtained in the study is depicted in the left panel of Fig. 2. In particular, the authors found that the position of the more evolved primary component in the Kiel diagram can only be explained when a large amount of extra mixing in terms of the overextension of the convective core (core overshoot $\alpha$ ) is included in the models. The system has been recently revisited by [31] based on high-quality space-based Kepler photometry and ground-based, highresolution HERMES [27] spectroscopy. The authors found that the evolutionary tracks computed for the dynamical masses of the stars fail to explain their positions in the Kiel diagram (see Fig. 2, right panel, solid lines), similar to the result obtained by [16] for the primary component. Either the mass/metallicity has to be changed to higher/lower value or an extra mixing had to be included in the models in terms of the core overshoot or rotational mixing to fit the components' positions in the Kiel diagram (dashed and dotted lines in the right panel of Fig. 2). An excess of mass, or the mass discrepancy, that was obtained by [31] amounts to about $10 \%$ which transforms into the age uncertainty of about $50 \%$. Since the mixing, mass, and metallicity correlate in the models and have similar effect on the tracks by shifting them towards higher temperature and extending towards lower gravities in the Kiel diagram, the problem can also be referred to just as "discrepant models". The term mass discrepancy is essentially used to quantify the level to which the models fail to explain high-quality observations of eclipsing binary stars.

The fact of evolutionary models lacking a certain amount of extra mixing is also asteroseismology proved. As it has been shown by [2], an extra mixing needs to be introduced in the terms of the core overshoot into the evolutionary models to match them with the observed seismic properties of binary components. However, it is barely the case for the majority of single stars of similar mass, where about the standard value of the core overshoot $\alpha=0.2$ is preferred, probably because the mass of a star can be way more relaxed in the modelling compared to the case of binary systems.

The same story seems to be repeating at early (pre-MS) stages of stellar evolution and at a lower mass end (below one solar mass). Recent studies of low-mass pre-MS stars based on K2 photometry [e.g., 11, 12, 20] show that the mass discrepancy is relevant for these objects as well and is even more pronounced (above $40 \%$ ) than in the case of high-mass main-sequence and giant stars. All three studies 

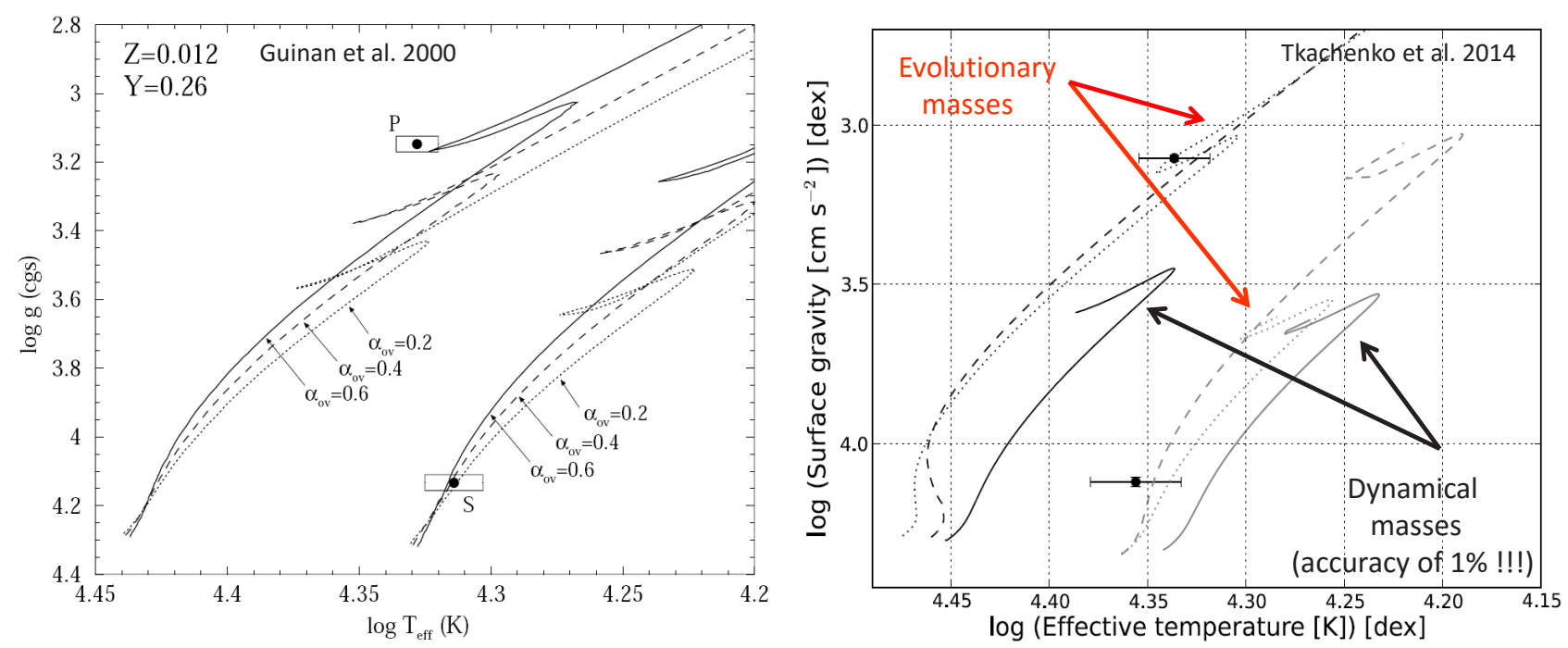

Figure 2: Position of both stellar components of the V380 Cyg system in the $\mathrm{T}_{\mathrm{eff}}-\log g$ Kiel diagram as obtained by [16] (left) and by [31] (right). The more evolved primary component has lower $\log g$ value and thus appears on top in both panels. The lines of different styles in the left panel represent evolutionary tracks computed assuming different values of the overshoot parameter (the exact values are indicated in the plot in the units of pressure scale height) and the (dynamical) masses obtained from the binary modelling. The solid lines in the right panel refer to the tracks computed for the dynamical masses of the stars and assuming standard value of overshoot of $\alpha=0.2$. The dashed and dotted lines represent the best fit evolutionary tracks obtained by including extra mixing into the models in terms of the overshoot and rotational mixing. See text for a discussion.

arrive to a similar conclusion: the models are not fully adequate as they generally tend to over-predict temperatures which is equal to under-predicting the masses. The problem seems to be mass-dependent and is more pronounced towards higher masses. The authors also found that different sets of evolutionary models are often inconsistent with each other. Moreover, the models are not internally consistent, which is nicely illustrated in Fig. 3. The plots show the positions of both stellar components of the UScoCTIO 5 binary system in the HR-diagram (top) and in the mass-radius diagram (bottom). The age obtained by fitting the isochrones to the stellar positions in the HR-diagram is a factor of two different from the one obtained by performing a similar fit in the observational mass-radius diagram. The authors find it very probable that the models fail to correctly predict the temperatures, possibly because they do not fully encompass the physics of energy transport (via convection and/or missing opacities). There is also a hint that incorporating the magnetic fields into the models might help to partially resolve the discrepancies. Magnetic fields inhibit the convection which results in decreasing the emergent flux and thus provides a slower contraction of a star during its pre-MS phase of evolution.

As it has been mentioned before, the mass discrepancy observed in binary stars is a manifestation of various shortcomings in the current theories/models of stellar structure and evolution. Thus, by studying in detail and resolving the mass discrepancy problem will help us to reveal the shortcomings in the theories and improve upon them. Observations wise, this requires an access to high-quality (space-based) photometric data which have to be complemented with the ground-based, high-resolution spectroscopy. Unfortunately, neither pre-MS nor massive stars have been represented in sufficient quantity in the field of view of the original Kepler mission, and only a few of them have been observed by CoRoT. However, many of these objects will be observed by the K2 and TESS missions, for the first time allowing us to approach the problem from statistical point of view, by analysing and charactiresing large star samples in a wide range of stellar masses.

\section{Rotationally induced mixing in high-mass stars}

The rotationally-induced mixing in high-mass stars is yet another topic that deserves a specific attention and is thus discussed here. The current theory predicts a significant enhancement of the nitrogen abundance at the surface of rapidly rotating stars, where the elements are transported by the rotational mixing to. [18] presented an observational confirmation of these theoretical predictions by finding a correlation between the surface nitrogen abundance and the projected rotational velocity from the analysis of a sample of 135 B-type stars in the Large Magellanic Cloud (see top panel of Fig. 4). However, the authors also reported on the detection of two groups of stars that contradict the theoretical predictions for the surface nitrogen enhancement due to the rotational mixing. These groups are termed "Group1" and "Group2" in the top panel of Fig. 4 and represent rapidly rotating stars with surface nitrogen abundances consistent with the LMC baseline composition and slowly rotating stars with enhanced surface nitrogen abundance, respectively. Assuming the theoretical predictions of the surface nitrogen enrichment are correct, these two groups of stars are of particular interest as there 

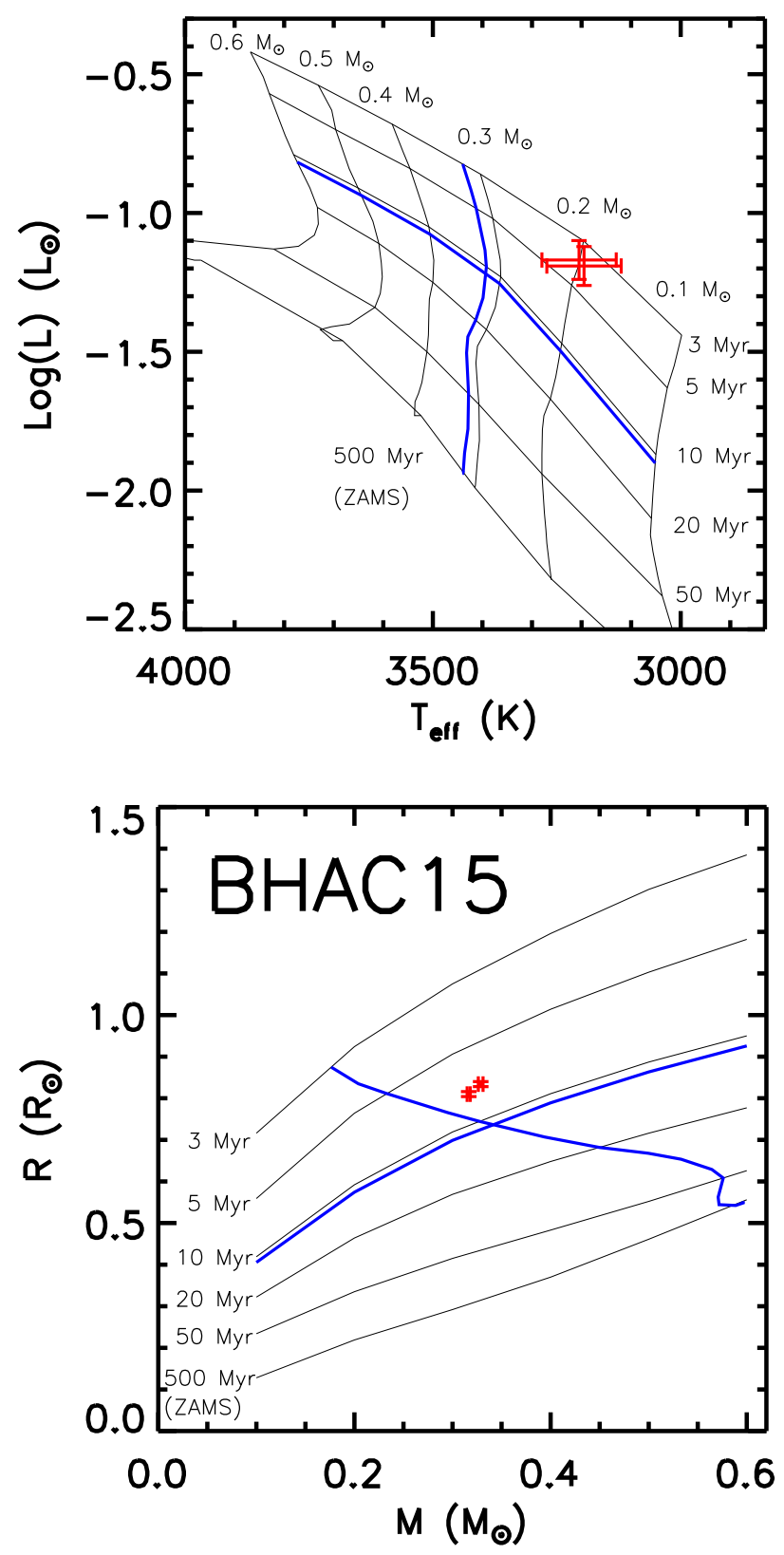

Figure 3: Positions of both stellar components of the UScoCTIO 5 binary system in the HR-diagram (top) and in the massradius diagram (bottom). The isochronal and isomass sequences are those predicted by the BHAC 15 models [6]. The figure is taken from [20].

must be some additional mechanisms involved (magnetic fields, binarity, etc.) that "pull out" the stars of the predicted correlation.

Although the correlation found by [18] indeed seems to be in line with the present theoretical predictions, neither of the two agree with the recent asteroseismic findings. In particular, [3] presented a detailed analysis of a sample of 70 B-type stars using multivariate statistical analysis techniques. Both the surface nitrogen abundance and the rotational frequency of the star has been included as the variables in the statistical analysis and, contrary to [18], the authors found that rotation has zero predictive
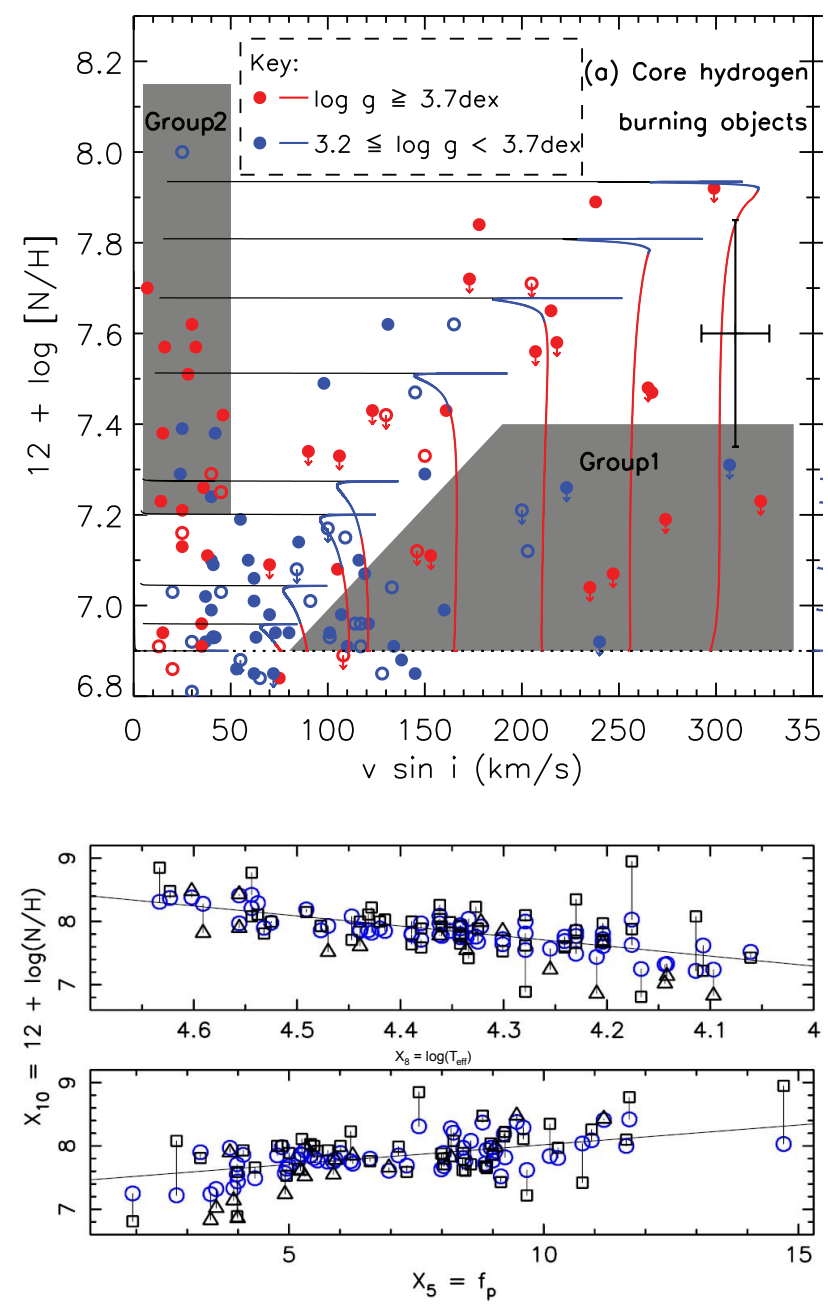

Figure 4: Top: Surface nitrogen abundance $(12+\log [\mathrm{N} / \mathrm{H}])$ versus the projected rotational velocity $(v \sin i)$ diagram for 135 B-type stars in the Large Magellanic Cloud. The typical error bars are indicated in the plot. Figure is taken from [18]. Bottom: Correlation of the surface nitrogen abundance with the effective temperature of the star and the frequency of its dominant acoustic oscillation mode. Figure has been adopted from [3].

power for the surface nitrogen abundance. Instead, the latter was found to correlate with the effective temperature of the star as well as with the frequency of its dominant acoustic oscillation mode (see bottom panel of Fig. 4). Thus, the largely observational asteroseismic analysis appears to be in contradiction with both the spectroscopic findings and the theoretical predictions as for the surface nitrogen enrichment driven by the rotational mixing in Btype stars.

Pulsating B-type stars in (double-lined spectroscopic eclipsing) binaries can help to unravel the ambiguity with the surface nitrogen abundance enrichment and its physical cause. Chemical composition analysis of B-type stellar components in binaries benefits greatly from the precisely determined values of the surface gravity (from dynamical mass and radius) and the effective temperature (net result of the removed degeneracy between $\log g$ and $\mathrm{T}_{\mathrm{eff}}$ ), allowing to reach a much higher precision in the individual 

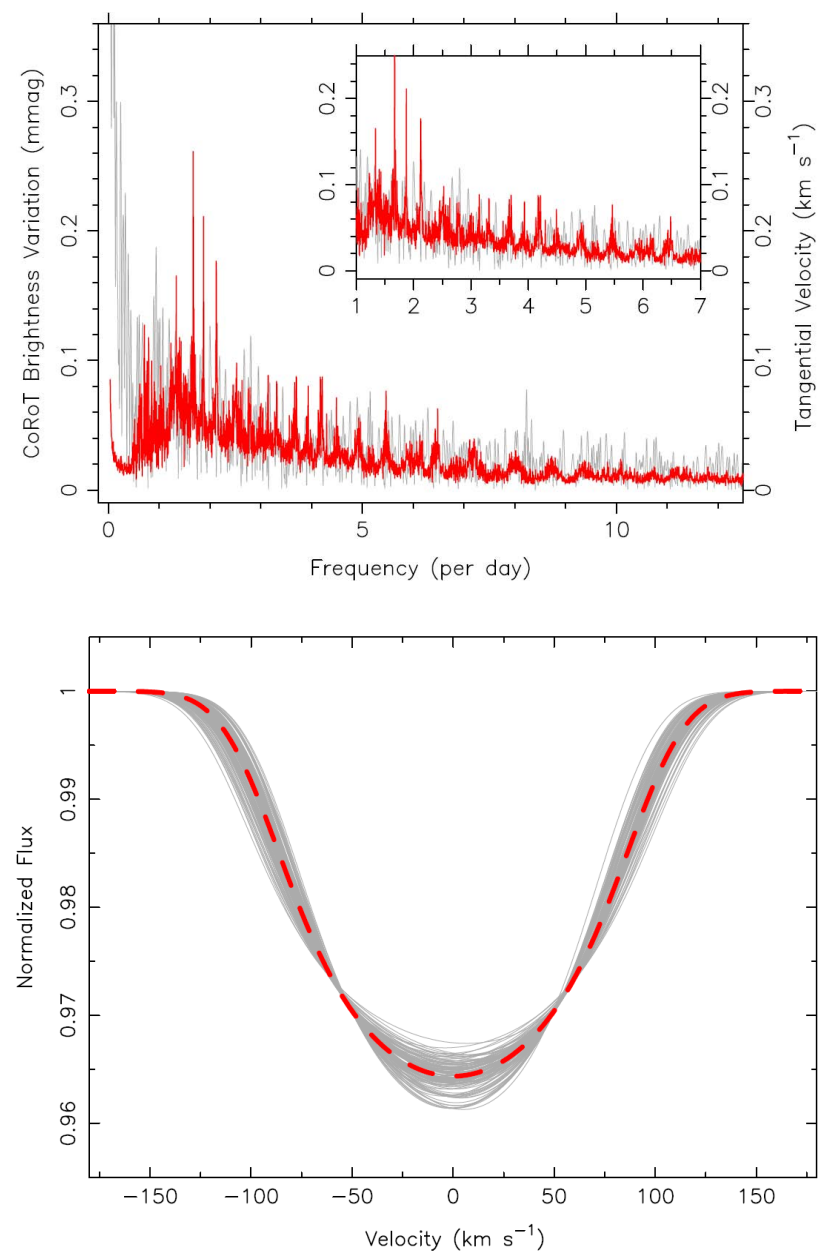

Figure 5: Top: Comparison of the amplitude spectrum constructed based on the 2D simulations of the IGWs (red) with the amplitude spectrum of HD 46150 observed by CoRoT (light grey). Bottom: Simulations of the line profile variations (light grey) caused by the IGWs in comparison with the line profile shape due to the rotational broadening only (red). Both plots has been adopted from [4].

abundance measurements than for single stars in the same mass/spectral type range. The results of such an analysis could be confronted with those obtained by [18] and [3] with the ultimate goal to 1) check whether any of the announced correlations are also applicable to binary stars, and 2) see whether the (possible) differences in the results can be attributed to the effect of the tides in binary systems.

\section{Internal gravity waves}

Another interesting phenomenon that is worth touching upon here is the Internal Gravity Waves (IGWs). The IGWs get excited in a stratified medium where the density decreases continuously or discontinuously with depth/height. In stars, these waves are generated at the boundary between the convective and radiative regions by convective motions. The IGWs has been studied theoretically and numerically [e.g., 21, 24, 28, 29, 32] quite extensively but essentially remained observationally undetectable until very recently. A quantitative comparison of the amplitude spectra constructed based on 2D simulations of IGWs with those observed by CoRoT have been performed by [4] for three O-type stars. Fig. 5 (top panel) illustrates a good overall agreement between the observed and simulated shapes of the Fourier spectrum of HD 46150, which is also representative of the other two stars studied, HD 46223 and HD 46966. The bottom panel of Fig. 5 illustrates the simulated line profile variations due to the surface velocity field generated by the IGWs. The variability is quite remarkable and appears to be consistent with additional line broadening observed in OB stars due to time-dependent macroturbulent velocity field.

The IGWs are also believed to be very efficient in transporting angular momentum and chemical species inside stars. Having said that, it is interesting to come back to the study performed by [18] for 135 B-type stars in the Large Magellanic Could, namely to the two groups of stars that were found to contradict the theory of rotationally induced mixing (see top panel of Fig. 4). The question to raise would be: could the existence of those two groups of stars be explained within the framework of IGWs? At first sight, the answer is probably "yes", assuming the IGWs can bring the angular momentum to the outer layers of a star as well as to extract it from them. The "Group1" stars (rapidly rotating stars with no surface nitrogen enrichment) could then be seen as intrinsically slow rotators in which the rotationally induced mixing is inefficient to bring nitrogen to the surface but whose outer layers are spun up by the IGWs leading to the detection of large $v \sin i$ values. And the other way around, the "Group2" stars would then be intrinsically rapid rotators whose outer layers got spun down due to the angular momentum extracted from them by the IGWs. However, this hypothesis still contradicts the asteroseismic findings by [2] who found no correlation between the surface nitrogen abundance and the rotational frequency/projected rotational velocity of the star based on the multivariate statistical analysis of a sample of 70 B-type stars. Nevertheless, the IGWs can still be (and probably are) largely involved in the transport of the chemical elements inside stars. Thus, it would be worth looking for a way to quantify the effect of the IGWs in massive stars and to include the corresponding parameter into the multivariate analysis similar to that performed by [2]. Should any correlation between the parameter describing the effect of the IGWs and the surface nitrogen abundance be found, this would point to the fact that the effect of the IGWs cannot be ignored when addressing the problem of the surface nitrogen enrichment in high-mass stars but must be taken into account along with the rotationally induced mixing.

Since the IGWs are probably generated in the majority of stars and are likely to play a major role in the transport of angular momentum inside them, it is also expected that these waves play a major role in the evolution of binary stars. The connection between the IGWs and the evolution of binary star systems in terms of circularisation of the orbit and synchronisation of the stellar components' spins are briefly touched upon in the next section. 


\section{Binary stars and tidal evolution theory}
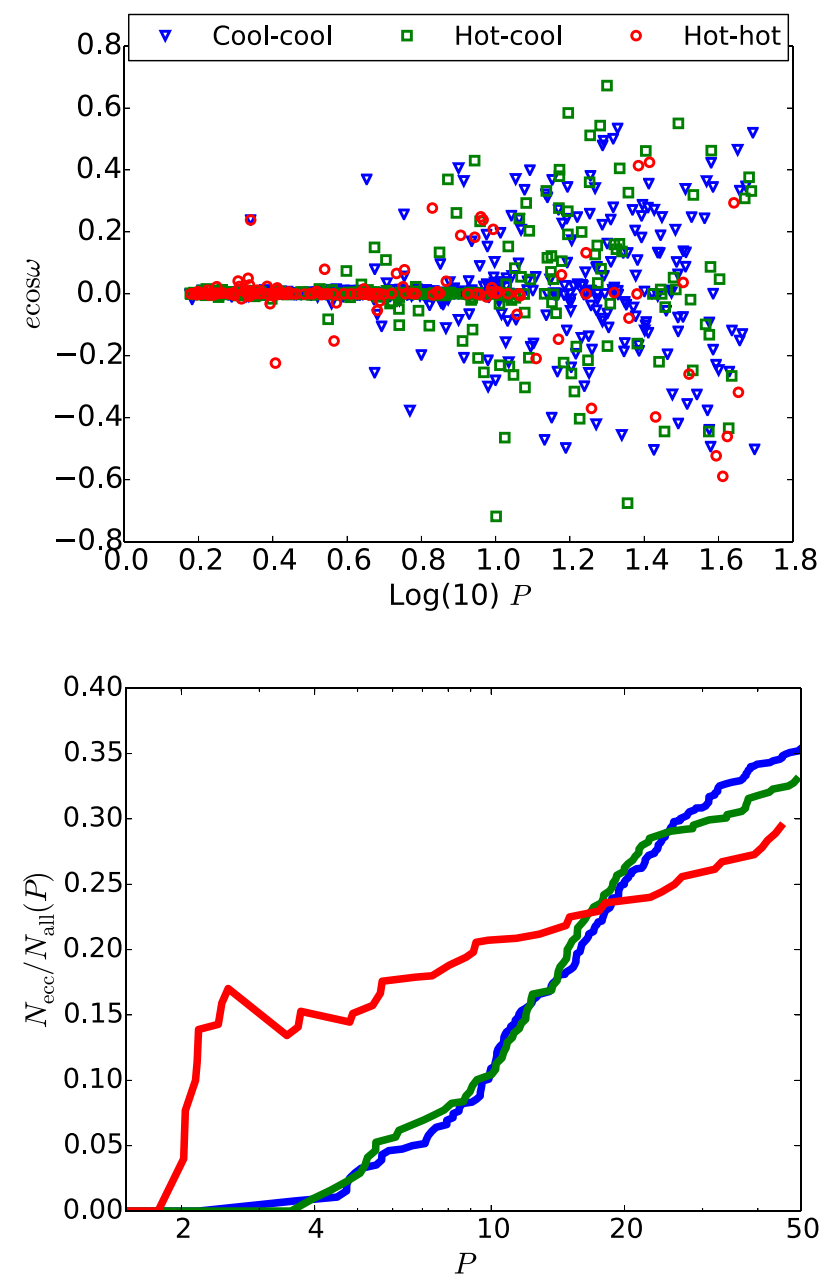

Figure 6: Top: A sample of some 900 Kepler binary systems on the $e \cos \omega-\log P$ diagram studied by [34]. The colours represent different system configurations in terms of the masses/spectral types of the individual stellar components: cool-cool (blue), hotcool (green), and hot-hot (red). Bottom: The cumulative fraction of eccentric systems as a function of the orbital period. The meaning of the colours is the same as in the top panel. Both plots have been adopted from [34].

The theory of tidal evolution describes the path that a binary star follows starting from its original configuration to the stage of lowest possible kinetic energy that is consistent with the conservation of angular momentum in the system. The lowest energy state is characterised by the alignment of the rotational axes of both stellar components with the normal to the orbital plane (also called spin-orbit alignment), circular orbit, and rotational periods of the stars equal to the orbital period of the system (also called spin-orbit synchronisation). Keeping it simple, the general pattern of the evolutionary path is as follows: in eccentric (close) binaries with individual components having non-synchronized spins, the tidal forces will raise differential motions in the stars, which will be dissipated into heat that is ultimately radiated into space. The excess energy comes at the expense of either the orbital energy or the spin energies of the stars, and a binary system tends to evolve into the state characterised by a circular orbit with the two stars having synchronised spins. Turbulent viscosity is one of the main dissipation mechanisms in stars with (large) outer convective zones [39-41], whereas damping of the resonances of the free pulsation g-modes by radiative dissipation is believed to be the dominant process in the tidal evolution for stars without (large) outer convective zones [42].

There is a number of issues with the current theoretical predictions concerning the synchronisation time scales. In particular, there are many examples in the literature where the individual stellar components exhibit either sub- or super-synchronous rotation while the theory predicts full synchronisation with the orbit. The situation is complicated by the fact that calculation of the spin-orbit synchronisation rates requires deep understanding of angular momentum transport inside individual stellar components. This is because the tidal torque that drives binary orbital evolution and synchronisation in particular is mostly applied to the outermost layers of the star, thus the synchronisation must be occurring from the stellar surface inwards. Looking at the effect of the IGWs in the context of spinorbit synchronisation is particularly interesting as these waves are very efficient in transporting angular momentum inside stars, as it has been mentioned above already. In fact, the IGWs may just be "disturbing" the individual stellar components of binary systems from their synchronous rotation by spinning up or down their outermost layers. This would make the stars to appear observationally nonsynchronised whereas they are in fact temporary pulled out of the spin-orbit synchronisation state by the IGWs transporting/redistributing angular momentum inside the stars. This hypothesis requires a further investigation which implies the observational detection of the IGWs in a wide range of stellar masses as well as the incorporation of the effect of these waves into the tidal evolution theory.

Contrary to the effect of the spin-orbit synchronisation, the current theory of tidal evolution performs reasonably well when it comes to the prediction of the orbital circularisation time scales. Recently, [34] investigated a sample of some 900 eclipsing binary stars observed by the original Kepler mission with the ultimate goal to confront the observations with the theoretical predictions concerning the circularisation time scales. Because of the lack of follow-up spectroscopic data, the parameter $e \cos \omega$ was used as a proxy for the eccentricity of the system. The sample was limited to stars with effective temperatures below $10000 \mathrm{~K}$, and three groups of binaries were defined according to the effective temperatures of the individual stellar components. These groups are: 1) coolcool, 2) cool-hot, and 3) hot-hot binaries, with hot being a star with the effective temperature above $6250 \mathrm{~K}$ (see top panel of Fig. 6). All stars were assumed to be on the main-sequence, so that the mass-radius-age relations could be used to estimate parameters necessary for the calculation of the circularisation time scales. The authors came to the conclusion that the theoretical predictions are in most cases in line with the observations, in particular for bi- 
naries containing at least one cool companion, although a few outliers were detected. Some cut-off periods were proposed beyond which the circularisation occurs at almost $100 \%$ rate, and it is worth noting that binaries with one cool and one hot component behave likewise the systems containing two cool stars (see bottom panel of Fig. 6). This result points to the fact that the tidal dissipation plays a major role in the observed distribution of the eccentricities for two groups of binaries with at least one cool component, whereas age is the dominant contributor for binaries formed of two hot stars.

Although very interesting and valuable by itself, the study of [34] can be extended/improved on several aspects. First, this concerns including more massive and hotter stars into the sample so that it also covers the entire spectral type B and thus binaries with more extreme mass ratios. Secondly, it would be worth including the spinorbit synchronisation aspect to quantify by how much the models fail to predict the corresponding time scales and to identify the mass-temperature regime that perhaps deserves a specific attention. Last but not least, it would be very beneficial for this type of the analysis to replace the many assumptions made with the observational constraints: replace the $e \cos \omega$ parameter by the eccentricity itself, feed the statistical analysis with the masses, radii, ages, etc. derived from binary dynamics and/or asteroseismology instead of assuming all stars being on the main-sequence and using the corresponding mass-radiusage relations, etc. The K2, TESS, and PLATO missions will provide a significant contribution to the problem as they cover a larger parameter space (in terms of stellar masses and evolutionary stages) than the original Kepler mission did. The TESS and PLATO missions will also target brighter stars which implies high-quality ground-based spectroscopic data can be obtained providing some of the missing observational constraints.

Despite the fact that the tidal evolution theory performs quiet well concerning the circularisation time scale predictions, the objects showing a remarkable disagreement between the theory and the observations are reported in the literature from time to time. The NGC 6791 turnoff binary system V568 Lyr is one of those objects where the observational measurements obtained from a combination of Kepler space-based photometry and ground-based high-resolution spectroscopy significantly disagree with the predictions of the tidal evolution theory. In particular, [38] found the system to be about 7.5 Gyr old and to have a circular orbit. Contrary to these findings, the tidal evolution theory predicts a significant eccentricity for the system and the tidal friction would need to be increased by a factor of $\sim 100$ in the models to make them consistent with the observations. This example clearly illustrates that there are cases where the theory also fails to predict the circularisation time scales, and such "outliers" are worth looking at to reveal the shortcomings in and improve upon the current theory of tidal evolution.

\section{Tidally-induced pulsations}

Tidally-induced pulsations in binary systems represent yet another physical mechanism that emphasises the importance of synergies between asteroseismology and binary star research fields. These are the gravity-mode free oscillations of stars that get resonantly driven by the dynamic tide in a binary system, typically during the periastron passage of the binary components where the tidal forces are the most efficient. As briefly discussed above, the tidallyinduced oscillations play a major role in the evolution of intermediate- to high-mass binary system as their radiative damping serves as the main energy dissipation mechanism in those objects. However, recent studies of intermediatemass binary systems observed by Kepler clearly showed that some of the resonantly driven modes are not damped in the radiative regions of stars but appear to have quite stable and large amplitudes so that they get observed at the stellar surfaces. The KOI-54 system discovered and observationally studied in detail by [37] is probably one of the most remarkable examples of pulsating binary stars where large fraction of the observed oscillation modes are resonantly driven by the tides. A distinct property of the tidally-induced pulsations is that they are observationally detected as exact harmonics of the orbital frequency in the Fourier spectrum, and the KOI-54 system exhibits the 90th and 91st harmonics as its largest amplitude oscillation modes. Several challenges have been raised by [37] concerning the pulsation properties of the KOI-54 system: 1) is it just one or both stars that are pulsating in the system?, 2) why do the 90th and 91st harmonics have the largest amplitudes?, and 3) what is the exact mechanism that feeds the observed pulsation modes and keeps them so stable in time, and why are not they damped in the radiative regions of a star? [13] investigated the KOI-54 system within the extended theory of tidal evolution with the ultimate goal to explain the observed oscillation properties of the star. The authors computed the predicted flux variations due the geometric and temperature effects of the modes and compared them to the observations of the two dominant modes in the system. Both observed oscillation modes could be explained by resonantly locked quadruple modes. The stability of these two modes over time is achieved through their resonant locking driven by the dynamical tides in the system and the intrinsic stellar spin down. The authors also show that the resonant locking provides an efficient way of transporting angular momentum from the orbit to the individual stellar components which implies that the KOI-54 system is going to evolve rapidly to zero eccentricity from the actual value of $\mathrm{e}=0.83$. Thus, the tidallyinduced pulsations represent an efficient way of transporting angular momentum within binary star systems and the space missions like Kepler, TESS, and PLATO provide us with the required high-quality observations to test and improve upon the current theories of tidal evolution.

\section{Scaling relations}

The scaling relations is the last topic that is discussed in this manuscript in the context of synergies between as- 


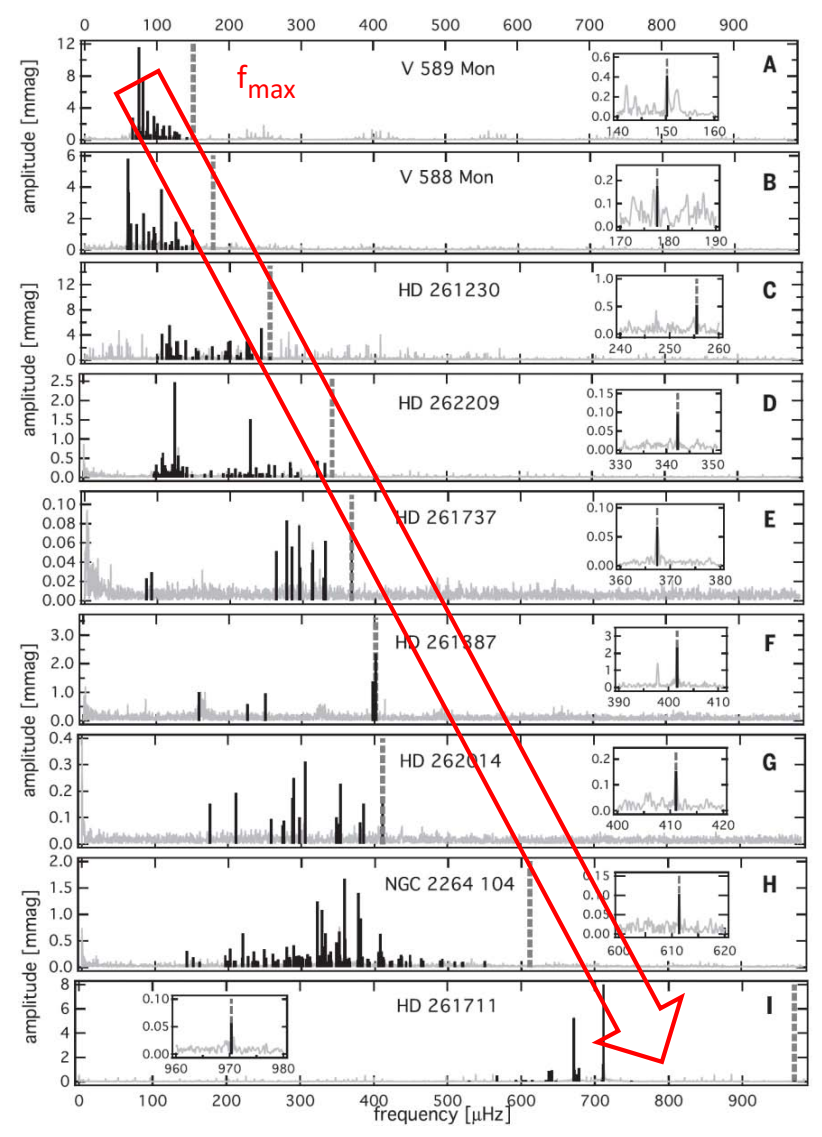

Figure 7: Correlation between the maximum pulsation frequency of the p-mode observed in a star and its relative age. The red arrow (schematically) shows how the pulsation properties of a star change as its moving towards the Zero Age Main Sequence (ZAMS). The plot has been adopted from [43].

teroseismology and binary stars. The scaling relations in asteroseismology stand for the relations between the fundamental properties of stars (masses and radii) and their seismic characteristics like large frequency separation $\Delta v$ and frequency of the maximum power $v_{\max }$. The relations hold for stars with extended convective envelopes that excite their pulsation modes stochastically and read

$$
\begin{aligned}
\frac{M}{M_{\odot}} & \simeq\left(\frac{v_{\max }}{v_{\max , \odot}}\right)^{3}\left(\frac{\Delta v}{\Delta v_{\odot}}\right)^{-4}\left(\frac{T_{\text {eff }}}{T_{\text {eff }, \odot}}\right)^{3 / 2} \\
\frac{R}{R_{\odot}} & \simeq\left(\frac{v_{\text {max }}}{v_{\text {max }, \odot}}\right)\left(\frac{\Delta v}{\Delta v_{\odot}}\right)^{-2}\left(\frac{T_{\text {eff }}}{T_{\text {eff }, \odot}}\right)^{1 / 2}
\end{aligned}
$$

Large frequency separation $\Delta v$ refers to the frequency spacing between the adjacent modes of stellar oscillation having the same degree $l$ (the total number of the surface nodal lines where no movement of the material occurs during the pulsation cycle) but different radial order $n$ (the total number of nodal lines inside the star in the radial direction). The relations were theoretically defined by [19] and are widely used nowadays to determine fundamental properties of stars pulsating in solar like oscillations. The relations assume that the properties of the star in question are close to those of the Sun which is often not the case (especially when the relations are applied to red gi-

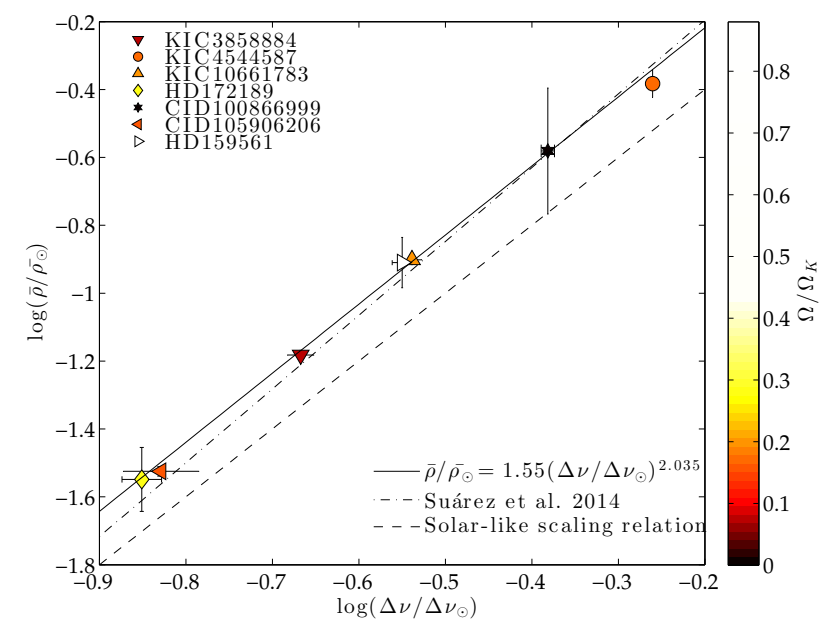

Figure 8: A relation between the mean density of the star and its large frequency separation in the low radial order regime for seven binary systems with main-sequence $\delta$ Sct type pulsating components. The solid line indicates the obtained empirical relation for MS $\delta$ Sct pulsators, the dashed line refers to the solar-like scaling relation, and the dashed-dotted line stands for the theoretically predicted relation for low radial order p-mode oscillations. The figure has been adopted from [14].

ants). For example, [23] pointed out that masses obtained from the scaling relations are on average accurate to about $10 \%$, which results in about $30 \%$ uncertainty on stellar age. [10] come to a similar conclusion, emphasizing that these uncertainties are due to the combined effects of models and adopted analysis methodology. The authors explicitly mention the need of more independent tests of the scaling relations, in particular tests of the estimated masses. The most obvious and precise way of tuning the scaling relations is to use the dynamical asteroseismology, which involves the study of solar-like pulsators in binary systems, where the fundamental, model-independent properties of stars inferred from binary dynamics can be confronted to the values obtained by means of the asteroseismic analysis. This field is currently being explored, in particular there was a talk given by Jean McKeever (see the corresponding contribution in these proceedings) in the session S10: Seismology: Stars Beyond the Main Sequence. The conclusions are essentially the same as those presented by [10, 23]: the relations perform quite well for the radius estimates whereas the masses are uncertain to about $10 \%$ level.

The idea of scaling relations can actually be extended towards the stars exhibiting heat-driven oscillations. However, instead of introducing theoretical relations, the synergies between asteroseismology and binary stars can be fully exploited to establish empirical relations between the fundamental properties of stars (obtained from binary dynamics) and their oscillation characteristics. For example, a clear relation between seismic properties and the age of a star has been found by [43] for pre-MS $\delta$ Sct type pulsators (see Fig. 7). The relation has been determined form the analysis of a sample of 9 pre-MS single stars and does not presently have a defined zero point, i.e. it allows to 
derive a relative age of a young star from its observed pmode pulsation properties. Detecting a $\delta$ Sct type pre-MS pulsator in a binary system would allow to put this relation on the absolute scale, which will certainly become possible in the near future with the K2, TESS, and PLATO observations.

[14] have studied seven binary systems containing at least one main-sequence $\delta$ Sct type pulsator. A clear relation between the mean density of the star (computed from the dynamical mass and radius) and the analog of the large frequency separation from solar case but in low radial order regime has been detected (see Fig. 8). The authors also found the empirical relation to be in a reasonable agreement with the theoretical relation computed for $\mathrm{p}$-mode oscillations in the low radial order regime.

The studies by [43] and [14] clearly demonstrate the potential of the dynamical asteroseismology in establishing empirical scaling relations between fundamental and seismic properties of stars exhibiting heat-driven oscillations. This opens a window for the determination of precise fundamental parameters of pulsating stars in wide range of stellar masses and evolutionary stages, thus providing critical observational input for testing and if necessary refining the current theories of stellar structure and evolution.

\section{Summary}

This paper touches upon just a few of many topics in stellar astrophysics that naturally arise from combining the research fields of asteroseismology and binary stars. The major points that have been discussed in the manuscript are summarized here:

- Many of the intermediate- to high-mass binary stars are found to exhibit the mass discrepancy problem which points to the shortcomings in the current theories of stellar structure and evolution. In the majority of cases, the inclusion of an extra mixing into the models in terms of the convective core overshoot allows to minimize the discrepancy between the dynamical and evolutionary masses of stars, but the high-overshoot values appear to be inconsistent with those seismically derived for single stars of similar masses and spectral type. Combining the fields of asteroseismology and binary stars will help us to reveal the shortcomings in and improve upon the current theories of stellar structure and evolution.

- The recent asteroseismic studies of B-type stars do not support the theoretical predictions and some of the spectroscopic findings concerning the rotationally induced mixing of chemical elements inside intermediate- to high-mass stars. Studying (pulsating) binary stars in similar mass/spectral type range would help to 1) significantly improve upon the accuracy of spectroscopically measured individual abundances, 2) explore whether there is a link between binarity and surface nitrogen enrichment (tidal effects, mergers, etc.), and 3) investigate a possible connection with the internal gravity waves excited in stellar interiors.
- Internal gravity waves are very efficient in transporting angular momentum inside stars. Moreover, these waves are also believed to play a major role in transporting chemical elements, thus might be an important physical ingredient in the surface nitrogen enrichment observed in B-type stars. Although the IGWs has been extensively studied theoretically in the past and both 2D and 3D numerical simulations are currently performed, the observational evidences of these waves are yet to be searched for and the effect is to be quantified using both space-based photometry and ground-based highresolution spectroscopy. The precise observational constraints will help us to improve upon the existing theories and to better understand the role of the IGWs in the evolution of single stars and multiple systems.

- The present-day theory of tidal evolution seems to be performing reasonably well when it comes to the prediction of the circularisation time scales in binary stars. However, the theory is still likely to be missing some important physical ingredients as it is often found to disagree with the observations of synchronisation rates of the individual stellar components. The highquality space-based photometric observations delivered by Kepler/K2, TESS, and PLATO and complemented with high-resolution ground-based spectroscopy open a window for studying new physical effects (tidallyinduced pulsations, IGWs, etc.) and incorporating them into the tidal evolution theory.

- Radiative damping of the resonantly driven oscillation modes is the main energy dissipation mechanism that drives the evolution of intermediate- to high-mass binary stars. However, the recent Kepler observations also show that some of those resonant modes get high and stable amplitudes instead of being damped. For the first time, we get the opportunity to confront the existing theories with the high-quality observations and to learn how the resonantly locked oscillation modes impact/change the evolutionary path of the individual binary components as well as the evolution of the system as a whole.

- The dynamical asteroseismology is a perfect tool for testing and if necessary refining the asteroseismic scaling relations for solar-like pulsators. It also holds a great potential for establishing empirical relations for stars of different masses and evolutionary stages that exhibit heat-driven oscillations.

\section{Acknowledgments}

The research leading to these results has received funding from the European Research Council (ERC) under the European Union's Horizon 2020 research and innovation programme (grant agreement $\mathrm{N}^{\circ}$ 670519: MAMSIE).

\section{References}

[1] Aerts, C., Christensen-Dalsgaard, J., \& Kurtz, D. W., Asteroseismology, (Astronomy and Astro- 
physics Library. ISBN 978-1-4020-5178-4. Springer Science+Business Media B.V., 2010)

[2] Aerts, C., EAS Publications Series, 64, 323 (2013)

[3] Aerts, C., Molenberghs, G., Kenward, M. G., \& Neiner, C., ApJ, 781, 88 (2014)

[4] Aerts, C., \& Rogers, T. M., ApJL, 806, L33 (2015)

[5] Auvergne, M., Bodin, P., Boisnard, L., et al., A\&A, 506, 411 (2009)

[6] Baraffe, I., Homeier, D., Allard, F., \& Chabrier, G., A\&A, 577, A42 (2015)

[7] Bedding, T. R., Mosser, B., Huber, D., et al., Nature, 471, 608 (2011)

[8] Bloemen, S., Hu, H., Aerts, C., et al., A\&A, 569, A123 (2014)

[9] Borucki, W. J., Koch, D., Basri, G., et al., Science, 327, 977 (2010)

[10] Chaplin, W. J., Basu, S., Huber, D., et al., ApJS, 210, 1 (2014)

[11] David, T. J., Conroy, K. E., Hillenbrand, L. A., et al., AJ, 151, 112 (2016a)

[12] David, T. J., Hillenbrand, L. A., Cody, A. M., Carpenter, J. M., \& Howard, A. W., ApJ, 816, 21 (2016b)

[13] Fuller, J., \& Lai, D., MNRAS, 420, 3126 (2012)

[14] García Hernández, A., Martín-Ruiz, S., Monteiro, M. J. P. F. G., et al., ApJL, 811, L29 (2015)

[15] Gillen, E., Aigrain, S., McQuillan, A., et al., A\&A, 562, AA50 (2014)

[16] Guinan, E. F., Ribas, I., Fitzpatrick, E. L., et al., ApJ, 544, 409-422 (2000)

[17] Herrero, A., Kudritzki, R. P., Vilchez, J. M., et al., A\&A, 261, 209 (1992)

[18] Hunter, I., Brott, I., Lennon, D. J., et al., ApJL, 676, L29 (2008)

[19] Kjeldsen, H., \& Bedding, T. R., A\&A, 293, 87 (1995)

[20] Kraus, A. L., Cody, A. M., Covey, K. R., et al., American Astronomical Society Meeting Abstracts, 227, 236.12 (2016)

[21] Kumar, P., Talon, S., \& Zahn, J., ApJ, 520, 859 (1999)
[22] Kurtz, D. W., Saio, H., Takata, M., et al., MNRAS, 444, 102 (2014)

[23] Miglio, A., Chiappini, C., Morel, T., et al., MNRAS, 429, 423 (2013)

[24] Montalban, J., \& Schatzman, E., A\&A, 354, 943 (2000)

[25] Nemec, J. M., Cohen, J. G., Ripepi, V., et al., ApJ, 773, 181 (2013)

[26] Pápics, P. I., Tkachenko, A., Aerts, C., et al., ApJL, 803, L25 (2015)

[27] Raskin, G., van Winckel, H., Hensberge, H., et al., A\&A, 526, A69 (2011)

[28] Rogers, T. M., \& MacGregor, K. B., MNRAS, 410, 946 (2011)

[29] Rogers, T. M., Lin, D. N. C., McElwaine, J. N., \& Lau, H. H. B., ApJ, 772, 21 (2013)

[30] Saio, H., Kurtz, D. W., Takata, M., et al., MNRAS, 447, 3264 (2015)

[31] Tkachenko, A., Degroote, P., Aerts, C., et al., MNRAS, 438, 3093 (2014)

[32] Townsend, A. A., JFM, 24, 307 (1966)

[33] Triana, S. A., Moravveji, E., Pápics, P. I., et al., ApJ, 810, 16 (2015)

[34] Van Eylen, V., Winn, J. N., \& Albrecht, S., ApJ, 824, 15 (2016)

[35] Van Reeth, T., Tkachenko, A., \& Aerts, C., A\&A, in press (2016, arXiv:1607.00820)

[36] Walker, G., Matthews, J., Kuschnig, R., et al., PASP, 115, 1023 (2003)

[37] Welsh, W. F., Orosz, J. A., Aerts, C., et al., ApJS, 197, 4 (2011)

[38] Yakut, K., Eggleton, P. P., Kalomeni, B., Tout, C. A., \& Eldridge, J. J., MNRAS, 453, 2937 (2015)

[39] Zahn, J.-P., Ann. Astrophysics, 29, 313 (1966a)

[40] Zahn, J.-P., Ann. Astrophysics, 29, 489 (1966b)

[41] Zahn, J.-P., Ann. Astrophysics, 29, 565 (1966c)

[42] Zahn, J.-P. 1975, A\&A, 41, 329-344

[43] Zwintz, K., Fossati, L., Ryabchikova, T., et al., Science, 345, 550 (2014) 